\title{
ATUAÇÃO DA ENFERMAGEM EM CIRURGIA CARDÍACA MINIMAMENTE INVASIVA VIDEOASSISTIDA*
}

\author{
Nursing practice in minimally invasive video-assisted cardiac surgery \\ Enfermería de práctica en cirugía cardiaca asistida por video mínimamente invasiva
}

Elisabete Silvana de Oliveira Sene', Dulcilene Pereira Jardim²

RESUMO: Objetivo: Relatar a atuação da enfermagem no perioperatório da cirurgia cardíaca minimamente invasiva videoassistida. Método: Trata-se de um relato de experiência da equipe de enfermagem no perioperatório dessa técnica cirúrgica, realizada em um hospital-escola que é referência em cardiologia no Estado de São Paulo. Resultados: Observa-se a importância da atuação da enfermagem no preparo do centro cirúrgico (CC), recepção do paciente, assistência ao ato anestésico, preparo do paciente para a cirurgia, atendimento à equipe no período intraoperatório e na saída do paciente de sala operatória (SO), atendendo às demandas desta modalidade cirúrgica inovadora. Conclusão: A enfermagem de centro cirúrgico (CC) deve ser capacitada a acompanhar a evolução das técnicas cirúrgicas, tanto no manuseio dos novos materiais e equipamentos como na assistência direta ao paciente, visando à segurança cirúrgica.

Palavras-chave: Enfermagem perioperatória. Enfermagem de centro cirúrgico. Cirurgia torácica videoassistida.

ABSTRACT: Objective: To report the nursing practice in the perioperative period of minimally invasive video-assisted heart surgery. Method: This is an account of the experiences of the nursing staff, during the perioperative period in this surgical technique, carried out in a teaching hospital that is a reference center for cardiology in the State of São Paulo. Results: The observation on the importance of the role of the nursing department in the preparation of the operating room, as well as in the reception of patients, assistance to anesthesia, preparation of patients for surgery, assistance to the team in the intraoperative period and when the patient leaves the operating room, and meeting the demands of this innovative surgical procedure. Conclusion: The nursing practice in the surgical center must be able to monitor the evolution of the surgical techniques, both in the handling of new materials and equipment and in direct patient care, aiming at surgical safety.

Keywords: Perioperative nursing. Operating room nursing. Thoracicsurgery,video-assisted.

RESUMEN: Objetivo: Presentar la práctica de enfermería en cirugía cardíaca mínimamente invasiva trans operativa videoassistida. Método: Se trata de un relato de la experiencia personal de enfermería durante la cirugía de esta técnica quirúrgica que se realiza en unos centros de referencia hospital universitario de São Paulo. Resultados: Toma nota de la importancia del papel de enfermería en la preparación de la sala de operaciones, así como en la recepción del paciente, la asistencia a la anestesia, la preparación del paciente para la cirugía, llame al equipo en el período intraoperatorio y la salida del paciente del sistema operativo, la satisfacción de las demandas de este procedimiento quirúrgico innovador. Conclusión: Un centro de Enfermería Cirurgico debe ser capaz de controlar la evolución de las técnicas quirúrgicas, tanto en el manejo de nuevos materiales y equipos como en la atención directa al paciente, el objetivo de la seguridad quirúrgica.

Palabras clave: Enfermería perioperatoria. Enfermería de quirófano. Cirugía torácica asistida por vídeo.

*Artigo produzido a partir do “Atuação da enfermagem em cirurgia cardíaca minimamente invasiva vídeoassistida”, Faculdade Israelita de Ciências em Saúde Albert Einstein (FICSAE), em 2015. 'Enfermeira. Discente do Curso de Pós-graduação em Enfermagem em Centro Cirúrgico, Recuperação Anestésica e Centro de Material e Esterilização (CC/RA/CME) da Faculdade Israelita de Ciências em Saúde Albert Einstein (FICSAE) - São Paulo (SP), Brasil. E-mail: betesene@hotmail.com

Rua Arraial de Santa Bárbara, 331 - Pedro José Nunes - CEP: 08061-360 - São Paulo (SP), Brasil.

${ }^{2}$ Enfermeira. Mestre em Ciências pela Universidade Federal de São Paulo (UNIFESP). Docente e Assistente de coordenação do Curso de Pós-graduação em Enfermagem em CC/RA/CME da FICSAE - São Paulo (SP), Brasil.

Recebido: 17 mar. 2016 - Aprovado: 16 ago. 2016

DOI: 10.5327/Z1414-4425201600030008 


\section{INTRODUÇÃO}

Historicamente, a cirurgia cardíaca tem pouco mais de seis décadas e, desde então, o progresso tem sido vertiginoso. A primeira cirurgia cardíaca bem-sucedida foi realizada em 1938, nos Estados Unidos da América (EUA), pelo Dr. Robert Edward Gross, para correção da persistência de canal arterial, e a primeira correção de defeito intracardíaco foi em 1952, pelo Dr. Floyd John Lewis, em uma paciente com comunicação interatrial. Em 1951, foi utilizada pela primeira vez a circulação extracorpórea (CEC) em humanos ${ }^{1}$.

A esternotomia mediana transesternal é o acesso mais utilizado para as operações cardiovasculares, tendo sido introduzida, em 1957, em substituição à toracotomia anterior bilateral, beneficiando um grande número de pacientes por reduzir o tempo de cirurgia, forneexcelente exposição do coração e diminuir o trauma respiratório, tornando-se o acesso de eleição mais utilizado nos dias de hoje. Porém, nos últimos anos, tem havido um grande interesse em diminuir o trauma cirúrgico e proporcionar maior conforto aos pacientes, o que deu início, em 2005, à nova fase da cirurgia cardíaca - a cirurgia minimamente invasiva, incluindo a cirurgia videoassistida, os procedimentos intracavitários através da CEC periférica, a assistência a vácuo e as minitoracotomias, sendo que o método cirúrgico empregado foi gradativamente progressivo, abrindo possibilidades para abordagens cirúrgicas a diversas cardiopatias valvares, obstruções coronarianas e defeitos congênitos ${ }^{2,3,4}$.

Conceitualmente, o termo "minimamente invasivo" refere-se a uma cirurgia realizada através de pequenas incisões, sem acesso direto ao coração ou outro órgão a ser operado, porém obtendo os mesmos resultados terapêuticos conseguidos com a cirurgia convencional ${ }^{4}$. Soma-se ainda a utilização da videoscopia na cirurgia minimamente invasiva, o que proporciona uma ampla visualização das cavidades intracavitárias em momentos pertinentes ao procedimento, garantindo êxito na técnica ${ }^{5,6}$.

Assim, a cirurgia cardíaca minimamente invasiva videoassistida (CCMIVA) mostra-se uma técnica segura com redução da dor e das complicações no pós-operatório, culminando com a diminuição da permanência hospitalar e consequente redução de custos de internação, além de maior satisfação estética do cliente decorrente da redução do trauma cirúrgico ${ }^{5,6}$.

Comparativamente, na cirurgia convencional por esternotomia mediana transesternal, ocorre a exposição ao ambiente de toda a cavidade intracardíaca, situação que, se prolongada, acarreta em aumento exponencial do risco de infecções e de desequilíbrio hidroeletrolítico, o que contribui para enfraquecer a resposta imunológica, resultando em maior tempo de permanência do paciente no hospital'

Diante de tantos avanços tecnológicos, novos desafios e responsabilidades surgem para a equipe de enfermagem do bloco cirúrgico. Portanto, toda a equipe necessita desenvolver treinamento específico para atuar de forma precisa nas cirurgias minimamente invasivas, tanto na assistência direta ao paciente como para acompanhar a rápida evolução dos equipamentos e dispositivos utilizados ${ }^{7}$. Assim, mostra-se importante o compartilhamento de experiências vivenciadas pela enfermagem do centro cirúrgico (CC) nesse tipo de procedimento, visando contribuir para o crescimento de outros profissionais e melhorar a assistência de enfermagem perioperatória.

\section{OBJETIVO}

Relatar a atuação da equipe de enfermagem no perioperatório de CCMIVA em um hospital-escola considerado referência em cardiologia do Estado de São Paulo.

\section{MÉTODO}

Trata-se de pesquisa descritiva do tipo relato de experiência, que tem interesse em estudar um fenômeno desconhecido ou pouco conhecido pela sociedade, explorando dados para construir um cenário. A pesquisa baseou-se na premissa de que os problemas podem ser resolvidos e as práticas podem ser melhoradas por meio de descrição e análise de observações objetivas e diretas ${ }^{8}$.

O estudo teve o intuito de descrever a experiência da pesquisadora e sua equipe no perioperatório de pacientes submetidos à CCMIVA em um hospital-escola referência em cardiologia situado na cidade de São Paulo. Essa instituição de grande porte possui 3 edificações com capacidade instalada de 373 leitos, sendo considerada um dos maiores hospitais de cardiologia da América Latina.

A divisão cirúrgica está organizada para atender à alta demanda institucional: dez cirurgias cardíacas convencionais e cinco cirurgias vasculares periféricas diárias são realizadas em oito salas cirúrgicas e uma sala para procedimentos híbridos, além de quatro leitos para recuperação anestésica (RA), utilizados basicamente para recuperação de pacientes 
submetidos a procedimentos híbridos de pequeno porte, como dissecção carotídea para via de acesso de cateterismo cardíaco e procedimentos percutâneos sem indicação de recuperação na unidade de terapia intensiva (UTI). A UTI conta com 50 leitos para recuperações pós-cirúrgicas e póstransplantes cardíacos.

O quadro de profissionais de enfermagem que atuam diretamente na assistência no CC é composto por 2 enfermeiras assistenciais no plantão matutino e 2 no plantão vespertino, 13 técnicos de enfermagem no período matutino e 11 no vespertino. Os plantões noturnos contam com seis técnicos e um enfermeiro em cada um. Os procedimentos cirúrgicos eletivos são realizados apenas no período diurno, tendo no noturno apenas cirurgias de emergência. Há, ainda, 12 instrumentadores cirúrgicos ( 6 no plantão matutino e 6 no plantão vespertino).

Em outra edificação da instituição, há mais quatro salas cirúrgicas destinadas a procedimentos como implantação de marca-passo, cirurgias vasculares periféricas e transplantes renais. Nesse mesmo prédio está sendo construída uma quinta sala cirúrgica, com o objetivo de implantar o centro de medicina fetal, que contará com 15 leitos de UTI neonatal e 10 leitos de UTI pediátrica.

São realizados, em média, 350 procedimentos cirúrgicos mensais, sendo que a CCMIVA, especificamente, tem apenas um procedimento mensal desde novembro de 2014, atualmente realizado pela mesma equipe cirúrgica.

\section{RESULTADOS}

As principais CCMIVAs realizadas na instituição do estudo são o implante de prótese mitral por prótese biológica ou mecânica e a plastia da valva mitral; em alguns casos, é realizado o implante de eletrodos epicárdicos.

Neste relato de experiência, foi apresentada a atuação da enfermagem perioperatória no implante de bioprótese de pericárdio mitral em suas atividades isoladas, bem como nas compartilhadas com os demais profissionais atuantes em sala operatória (SO), como o anestesista, o cirurgião principal, o cirurgião assistente, o perfusionista, o instrumentador cirúrgico, o técnico da engenharia clínica e o médico ecocardiografista.

Por ser um procedimento cirúrgico específico e inovador, foi necessário o preparo da equipe de enfermagem para atuação sistematizada com o intuito de prevenir riscos ao paciente inerentes ao próprio procedimento cirúrgico, seguindo os passos descritos a seguir, cujas ações tiveram como bases norteadoras o ensino, a gestão e a assistência.

Na temática do ensino, buscou-se assegurar a manipulação correta dos novos dispositivos e equipamentos utilizados na CCMIVA. Foi realizado um treinamento com aulas práticas pela empresa responsável, englobando a equipe de enfermagem do CC, centro de material e esterilização (CME) e os instrumentadores cirúrgicos.

No que tange à gestão, foi realizada a devida previsão e provisão dos materiais e equipamentos necessários à realização da CCMIVA, além da padronização de itens para a execução do procedimento cirúrgico, com a criação de checklists utilizados na SO e impressos de requisição de materiais específicos. Tendo em vista a segurança do paciente, foram criados protocolos institucionais referentes à assistência ao paciente no período intraoperatório.

Referente à assistência, o Quadro 1 mostra a sequência de ações e procedimentos realizados pela equipe multiprofissional, o que inclui a enfermagem de CC, para a realização do procedimento de implante de bioprótese pericárdio mitral minimamente invasiva e videoassistida (Figura 1).

\section{DISCUSSÃo}

A introdução de novos procedimentos cirúrgicos considerados inovadores como a CCMIVA deu-se em resposta à necessidade de menores incisões, mas com visualização ampliada do campo operatório, proporcionando os mesmos resultados obtidos ao longo dos anos com a cirurgia realizada de maneira convencional, agregando vantagens perceptíveis no quadro clínico do paciente nos períodos intra- e pós-operatório.

A técnica exige adaptação do cirurgião ao abordar as cavidades cardíacas de forma restrita pelo comprimento limitado da incisão, o que é compensado pelo uso da videotoracoscopia. O manuseio de instrumentos longos e a visibilidade indireta do campo operatório são dificuldades superadas gradativamente pela sucessão de intervenções cirúrgicas, através da qual as habilidades e destrezas nas manipulações são aperfeiçoadas ${ }^{10}$.

Da mesma forma, o procedimento representa um desafio para a equipe multiprofissional, o que inclui a enfermagem do CC, visto que exige da enfermeira assistencial e do circulante de sala adaptação às necessidades da equipe e do paciente no período transoperatório.

Nesse sentido, é indispensável que a equipe de enfermagem envolvida na CCMIVA receba treinamento adequado 
Quadro 1. Sequência de procedimentos para a realização da cirurgia cardíaca minimamente invasiva videoassistida em hospitalescola cardiológico no Estado de São Paulo.

\section{Indicação cirúrgica}

Os critérios que indicam a possibilidade de realização da CCMIVA na instituição incluem: pacientes eletivos, sem cirurgia cardíaca ou torácica prévia, peso acima de 50 kg e fração de ejeção acima de $50 \%$.

Critérios de exclusão: pacientes obesos, dilatação da aorta ascendente e deformidades torácicas (pectus excavatum e pectus carinatum).

A decisão pelo procedimento é tomada pelo médico cardiologista clínico juntamente ao cirurgião cardíaco, com os devidos esclarecimentos ao paciente e sua família. Posteriormente, é realizado o preenchimento do aviso de cirurgia que é encaminhado ao CC pelo cardiologista clínico para o agendamento da cirurgia.

\section{Agendamento cirúrgico}

È feito diretamente no CC pelo cirurgião principal e assistente, após análise do histórico do paciente e exames complementares pré-operatórios (eletrocardiograma, radiografia de tórax, ecocardiograma transesofágico, ultrassom de artérias carótidas, aorta abdominal, ilíacas e femurais), por meio do preenchimento do impresso específico, no qual descreve os dados do paciente, cirurgia e equipe, além dos materiais e equipamentos necessários para o procedimento cirúrgico. 0 impresso é entregue posteriormente à enfermeira do $\mathrm{CC}$, responsável pelo mapa cirúrgico, que prevê e provê os mesmos junto à farmácia, CME e almoxarifado para o dia da cirurgia.

\section{Preparo da sala operatória}

Na data programada para a cirurgia, a enfermeira assistencial confere a existência de itens como reservas de hemoderivados, leito disponível na UTI, além de realizar junto ao circulante o checklist institucional de montagem da SO. Este checklist verifica a presença e bom funcionamento de itens gerais como limpeza preparatória, presença de equipamentos, carro de consumo de materiais, carro de medicamentos para anestesista, materiais necessários para intubação orotraqueal e mobiliário, além de materiais e equipamentos permanentes e consignados específicos da CCMIVA solicitados no agendamento. 0 técnico da engenharia clínica realiza o teste de todos os dos equipamentos em SO antes da entrada do paciente.

\section{Recepção do paciente no CC}

0 encaminhamento do paciente para o CC é realizado em maca acompanhado por familiares e pela enfermagem da unidade de internação. Na entrada do CC, a enfermeira, o recepciona com o cirurgião assistente, checam e confirmam os seguintes dados: nome completo, pulseira de identificação, prontuário e preenchimento termo de consentimento anestésico e cirúrgico. Ambos esclarecem possíveis dúvidas ainda existentes tanto pelo paciente como pelos familiares e o enfermeiro faz o encaminhamento do paciente à SO.

\section{Recepção do paciente em So}

Na SO, o paciente é recepcionado pelo circulante de sala, que, com o auxílio da enfermeira, realiza sua transferência para a mesa cirúrgica sob uma manta térmica aquecida a $38^{\circ} \mathrm{C}$, em posição dorsal horizontal. Nesse período, o ar-condicionado da sala de cirurgia permanece desligado.

0 circulante de sala confere novamente a pulseira de identificação, prontuário e termos de consentimentos devidamente assinados. A enfermeira realiza o preenchimento do impresso da SAEP, compreendendo admissão em sala e prescrevendo através de checklist as intervenções de enfermagem a serem realizadas durante a cirurgia, bem como os itens de segurança cirúrgica, o que é feito em impresso próprio. Todos os itens prescritos são checados pelo circulante durante a cirurgia.

\section{Preparo da mesa cirúrgica}

Paralelamente, o instrumentador cirúrgico revisa a presença de materiais estéreis de uso único, materiais permanentes e consignados (próteses, cânulas arteriais e venosas, fios de neocorda de PTFE — fio cirúrgico de politetrafluoretileno, Goretex e anéis rígido-Carpentier). Após a conferência, o instrumentador se paramenta e inicia a montagem da mesa cirúrgica com o auxílio do circulante da sala, que dispõe, de forma asséptica, os materiais previamente separados e confere os integradores, que são armazenados em impresso próprio no prontuário do paciente.

\section{Preparo do paciente ao ato anestésico}

Com o paciente em mesa cirúrgica, é realizada a monitorização não invasiva pela enfermeira (eletrodos, pressão arterial invasiva e oxímetro de pulso), seguida da punção venosa pelo anestesista em antebraço direito. 0 paciente é posicionado sentado para a realização da raquianestesia, seguida da colocação das pás descartáveis para desfibrilação cardíaca externa, colocadas em região escápular direita e região anterolateral do hemitórax esquerdo, bem como a inserção da placa descartável de bisturi elétrico em região lombar direita. 0 paciente é novamente posicionado em decúbito dorsal horizontal o eletrodo de eletroencefalograma contínuo é colocado em região frontal para monitorização da dinâmica cerebral durante a anestesia. Inicia-se a indução anestésica por via inalatória e venosa, procedendo à intubação orotraqueal, fixação do tubo, proteção ocular, passagem do sensor de temperatura esofágica e sonda do ecocardiograma. 
Quadro 1. Continuação.

Preparo do paciente

Após a realização da anestesia, a roupa do paciente é retirada, respeitando sua privacidade e conforto, possibilitando a checagem detalhada da integridade da pele, higiene corporal e tricotomia (tórax anterior e posterior, região axilar e inguinal direita e esquerda), a qual é feita no quarto imediatamente antes do paciente ser encaminhado para o CC.

0 cirurgião assistente se paramenta para realização da punção venosa central e arterial, seguindo o protocolo da instituição para a posição dos acessos - lado direito para acesso periférico (antebraço) e acesso central duplo lúmen (subclávia) para administração das drogas vasoativas, anestésicos e para mensurar a PVC; e lado esquerdo para o controle da PAM e controle da gasometria arterial. Após esses procedimentos, é realizada a passagem de cateter vesical de demora para controle do débito urinário.

0 posicionamento dorsal horizontal é mantido, porém com a colocação de coxim sob a região lombar direita, para garantir um ângulo inclinação de $30^{\circ}$, sendo os braços mantidos e fixados na extensão do corpo. 0 cirurgião principal faz as demarcações incisionais da minitoracotomia e 0 assistente procede à degermação (solução de clorexidina degermante) e à antissepsia da pele do paciente (solução de clorexidina alcoólica). É realizada a colocação dos campos cirúrgicos pela equipe já paramentada, deixando a região do tórax exposta, assim como a inguinal direita, para instalar as cânulas da CEC.

\section{0 intraoperatório: CCMIVA}

A mesa de instrumental e mesa auxiliar com todos os materiais dispostos são aproximadas e é feita a conferência do número de compressas grandes, pequenas e gases pelo instrumentador, com devida anotação pelo circulante de sala em impresso específico. A seguir, são posicionadas pelo técnico da engenharia clínica, circulante de sala e enfermeiro as duas estantes de vídeo, bisturi elétrico, desfibrilador, hampers, além da abertura dos materiais consignados e outros específicos (caixa com a fibra ótica e caixas para cirurgia minimamente invasiva) após conferência pelo enfermeiro.

É colocada sobre a pele do paciente um filme aderente e transparente a base de clorexidine para uma melhor fixação dos campos cirúrgicos e proteção do campo operatório. 0 cirurgião principal inicia as incisões de $4 \mathrm{~cm}$, ao nível do $4^{\circ}$ EICD, entre a LAA e a LHD (Figura 1). São aproximadas a máquina de CEC e o aparelho de ecocardiograma, e a primeira amostra de sangue arterial é analisada para controle dos parâmetros sanguíneos do paciente.

Após a heparinização plena do paciente, a CEC é estabelecida por meio de canulação femoral à direita e a temperatura é mantida em $30^{\circ}$. Segue-se o ato cirúrgico propriamente dito com pinçamento aórtico, atriotomia esquerda, excisão valvar, passagem dos pontos pelo anel valvar, assentamento da prótese biológica e os nós com os fios cirúrgicos, Utilizando-se a visibilidade exclusiva pelo monitor de vídeo. Durante o procedimento, é realizado revezamento do posicionamento da mesa cirúrgica em Trendelemburg, Trendelemburg reverso, lateral direita e esquerda pelo anestesista. No período de CEC, os controles dos valores de gasometria arterial, hematócrito, hemoglobina, eletrólitos e TTPA são verificados a cada 30 minutos pelo perfusionista e pelo anestesista, permitindo realizar as correções necessárias. Os exames são encaminhados e os resultados recebidos também pela enfermagem, além da conferência de hemoderivados, quando necessário.

Finalizado o procedimento cirúrgico, é realizada uma inspeção das cavidades com o auxílio da ecocardiografia transesofágica e o circulante de sala auxilia no reaquecimento do paciente por meio da manta térmica a $38^{\circ} \mathrm{C}$, infusões venosas aquecidas e desligando o ar-condicionado. Para retorno do coração em ritmo sinusal, o cirurgião solicita ao circulante de sala o preparo de uma carga de desfibrilação externa de 10 joules.

É realizada a conferência ativa e conjunta das perdas sanguíneas por compressas e gases e balanço hídrico (registradas em impresso próprio), além do correto armazenamento de material retirado do paciente para posterior encaminhamento à anatomia patológica, devidamente protocolada pela enfermagem.

Os curativos e drenos são instalados com introdução de solução fisiológica $0,9 \%$ nos frascos para drenagem de ar e sangue da cavidade. 0 circulante de sala demarca externamente no frasco o volume em $\mathrm{ml}$ e a hora para controle.

A enfermagem desliga os equipamentos, afastando-os para retirada dos campos cirúrgicos, com verificação da inexistência de instrumentais perdidos, bem como verificação da integridade da pele do paciente, especialmente nas regiões dorsal, sacro-glútea e calcâneos, finalizando o preenchimento do SAEP — diagnósticos de enfermagem e as intervenções necessárias no pós-operatório imediato.

Saída do paciente de So

A enfermeira realiza por telefone a passagem do plantão para a enfermeira da UTI com os seguintes dados: o nome e idade do paciente, anestesia e cirurgia realizada, posições e condições de cateter venoso periférico, central e arterial, drenos, curativos e infusões medicamentosas, bem como intercorrências.

Realiza ainda a conferência do correto preenchimento das anotações de enfermagem pertinentes ao período intraoperatório, além do preenchimento dos cuidados pós-operatórios, bem como os demais impressos do prontuário de responsabilidade do anestesista, perfusionista e cirurgião. Recolhe material consignado utilizado e não utilizado na cirurgia, entregando-os na farmácia.

É realizada a transferência do paciente da mesa cirúrgica para cama com auxílio do transfer, e o monitor de transporte portátil multiparâmetros e oxigênio suplementar são posicionados. As infusões medicamentosas são identificadas, drenos e outros dispositivos são reorganizados e o paciente é coberto. 0 transporte do paciente para a UTI é acompanhado pelo anestesiologista, pelo cirurgião assistente e pelo circulante de sala.

0 cirurgião principal atende aos familiares em sala anexa ao CC para informações sobre o intra e o pós-operatório do paciente.

CEC: circulação extracorpórea; CC: centro cirúrgico; SO: sala operatória; PVC: pressão venosa central; PAM: pressão arterial média; EICD: espaço intercostal direito; LAA: linha axilar anterior; LHD: linha hemiclavicular direita; TTPA: tempo de tromboplastina parcial ativa; UTI: unidade de terapia intensiva; SAEP: Sistematização da Assistência de Enfermagem Perioperatória. 


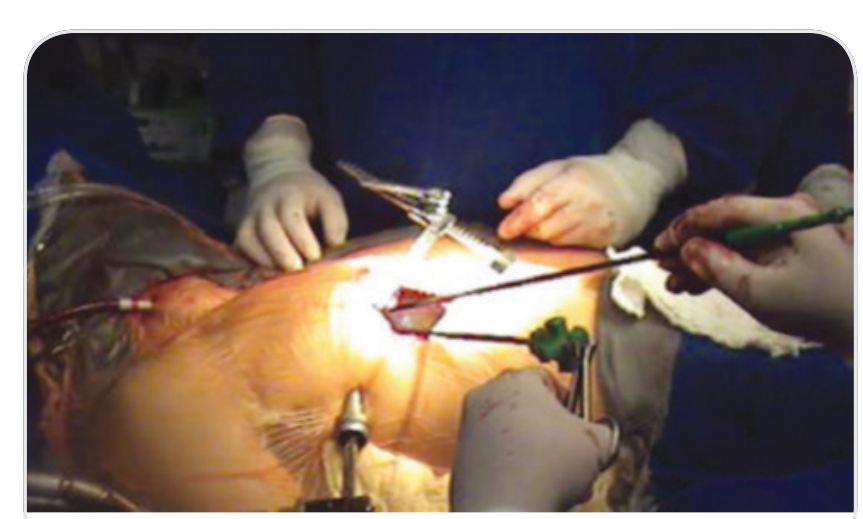

Fonte: Fortunato?

Figura 1. Visão panorâmica da minitoracotomia lateral direita para acesso à valva mitral.

para o desempenho de suas ações com conhecimento e segurança, tanto em atividades assistenciais como no manuseio dos dispositivos e equipamentos específicos desse procedimento, visando à segurança do paciente durante todo o procedimento anestésico-cirúrgico.

Para facilitar o correto atendimento da equipe cirúrgica e do próprio paciente na CCMIVA, é necessária a previsão e provisão desses materiais e equipamentos, além de sua padronização por meio da criação de checklist com itens mínimos necessários para realização do procedimento, de forma a facilitar a montagem da SO pelo circulante de sala e a checagem pela enfermeira antes da entrada do paciente em sala cirúrgica ${ }^{11}$.

A padronização é uma importante ferramenta gerencial e, como tal, confere uniformidade às ações, além de reduzir a dispersão e possibilitar que cada profissional realize seus serviços de forma orientada e segura ${ }^{12}$.

Com uma equipe bem treinada e com os materiais e equipamentos disponíveis na SO, a assistência de enfermagem perioperatória será mais efetiva. Associam-se a isso medidas de suma importância, como a aplicação da lista de verificação de segurança cirúrgica (checklist), que consta das etapas: Sign in - antes da indução anestésica; Timeout - antes da incisão na pele; e Sign out - ao término da cirurgia, antes do paciente sair da SO. Essas ações são realizadas por um coordenador da lista de verificação, frequentemente um profissional de enfermagem, visando à segurança do paciente ${ }^{11,13}$.

Para o sucesso do ato anestésico-cirúrgico, é fundamental a participação ativa do enfermeiro em todo o período transoperatório, atuando diretamente desde a recepção do paciente no CC até sua saída da SO, com cuidado individualizado e humanizado ${ }^{14}$.
É recomendável que a recepção do paciente no CC seja feita pelo enfermeiro, de preferência pelo mesmo profissional que fez a visita pré-operatória de enfermagem, para que este dê continuidade à aplicação do Sistema de Assistência de Enfermagem Perioperatória (SAEP), além do fato de esse profissional já ser conhecido pelo paciente, o que pode lhe trazer mais segurança. Da mesma forma, a recepção do paciente na SO deve ser realizada, preferencialmente, pelo mesmo profissional que o recebeu no CC, realizando-se a apresentação do ambiente e da equipe ${ }^{14}$.

O auxílio ao ato anestésico deve ser feito prioritariamente pelo enfermeiro, cujo preparo é essencial no atendimento às situações de emergência, paralelamente ao preparo da mesa cirúrgica realizado pelo instrumentador cirúrgico com o auxílio do circulante de sala, bem como o preparo da equipe cirúrgica.

$\mathrm{O}$ anestesista introduz no paciente uma sonda de ecocardiograma, pois, na CCMIVA, a utilização de ecocardiografia esofágica nos períodos pré e intraoperatório é considerada fundamental, a fim de identificar os mecanismos de insuficiência valvar, bem como assegurar que toda a cavidade intracavitária esteja sem ar residual na saída de CEC. Desde o início do uso da técnica na instituição deste estudo, a ecocardiografia transesofágica é considerada padrão em todo procedimento por vídeo, visando oferecer segurança e eficácia ao procedimento cirúrgico.

O enfermeiro deve participar no posicionamento cirúrgico tanto para a realização do ato anestésico como para o ato cirúrgico, sendo essa uma ação compartilhada pela equipe com foco na segurança do paciente e na identificação de riscos para prevenção da formação de úlceras por pressão em cirurgias de grande porte com predominância da posição de decúbito dorsal horizontal (DDH), em função do tempo de duração e mobilização. A anestesia geral propicia maior ausência de sensibilidade, e por ser utilizada por períodos mais prolongados, como nas cirurgias cardíacas, prejudica a troca de oxigênio e gás carbônico, pois o organismo perde os mecanismos de compensação, ficando susceptível ao desenvolvimento de lesões ${ }^{15}$.

A utilização da eletrocirurgia requer cuidados especiais da equipe. Recomenda-se que a placa dispersiva seja colocada após o posicionamento cirúrgico, próximo à incisão cirúrgica, sobre a pele limpa, seca, sem pelos, sobre a massa muscular e em região bem vascularizada, não sendo preconizado um local específico. Ao término do procedimento, a remoção da placa dispersiva deve ser realizada com cuidado e observação de possíveis lesões, atentando aos registros de enfermagem relacionados com o uso do equipamento ${ }^{16}$.

O preparo do paciente cirúrgico inclui ainda a realização de técnicas como tricotomia, degermação de pele e sondagem vesical de demora (SVD), que podem ser executadas pelo 
enfermeiro na SO. Especificamente na instituição do estudo, a tricotomia é realizada no quarto imediatamente antes do encaminhamento do paciente para o CC, e a degermação de pele bem como a SVD são realizadas pelo cirurgião assistente em sequência aos procedimentos de acessos centrais já descritos.

Quando a equipe e o paciente estão prontos, dá-se início à cirurgia, e a função do circulante de sala se desenvolve com mais tranquilidade no atendimento às necessidades intraoperatórias.

Na CCMIVA, a utilização da CEC tem função primordial para uma perfusão segura, com controle rigoroso dos fluxos sanguíneos, de oxigenação e de temperatura, realizado pelo perfusionista e anestesiologista por meio do controle dos parâmetros sanguíneos, trocas gasosas e volume sanguíneo para as correções necessárias ${ }^{17}$, conforme resultados dos exames encaminhados e trazidos pelo circulante de sala.

Nessa cirurgia, os tempos de CEC e pinçamento aórtico são mais longos quando comparados à técnica convencional via toracotomia mediana transesternal. Porém, é um procedimento seguro, associado a menor morbidade, dor no pós-operatório, sangramento e necessidade de transfusão sanguínea, tempo de extubação, permanência em UTI e internação hospitalar, com retorno às atividades cotidianas mais rapidamente ${ }^{18}$.

Finalizando a cirurgia, o circulante de sala e o enfermeiro realizam procedimentos como a conferência de compressas, a identificação de peças para anatomia patológica, o preenchimento de impressos e a organização do prontuário, a transferência do paciente para cama, o transporte e a passagem de plantão para UTI.Outro fator importante é que um membro da equipe cirúrgica providencie a comunicação com os familiares do paciente para informações sobre o procedimento, a situação e a localização do paciente.

Para procedimentos cirúrgicos de grande porte como as cirurgias cardíacas, é importante ainda que a sala cirúrgica possua dimensões físicas adequadas, devido ao número de equipamentos e profissionais envolvidos no procedimento, sendo preconizado que a SO possua um espaço físico de, pelo menos, $36 \mathrm{~m}^{2}$, com dimensão mínima de $5 \mathrm{~m}^{19}$, dimensões atendidas pelas salas cirúrgicas da instituição deste estudo.
Cabe acrescentar que a infecção do sítio cirúrgico (ISC) ainda é um dos fatores complicadores nas cirurgias cardíacas. Porém, dados estatísticos comprovam uma redução de 90\% no risco de ISC na CCMIVA quando comparada aos métodos convencionais das toracotomias medianas transesternais, cujo risco de apresentar infecção está acima de 30\%, com uma taxa de mortalidade de 10 a $47 \%{ }^{20}$.

Diante do exposto, é vital que todos os membros da equipe de enfermagem estejam cientes de seu papel e trabalhem em sintonia para garantir a qualidade da assistência prestada ao paciente cirúrgico, com constante avaliação da qualidade, prevenção e correção de possíveis erros no processo e reformulação da assistência, a fim de assegurar os direitos, a segurança e o bem-estar do paciente.

\section{CONSIDERAÇÕES FINAIS}

Com a constante evolução das cirurgias - entre elas, a cardíaca - , novas tecnologias vêm sendo desenvolvidas com o objetivo de tornar os procedimentos cirúrgicos cada vez mais seguros e menos invasivos, resultando em melhores resultados funcionais e estéticos.

As CCMIVAs, como os demais procedimentos cirúrgicos, requerem ativa participação da enfermagem perioperatória, desde o preparo do ambiente, a recepção do paciente, a assistência ao ato anestésico, o preparo do paciente para a cirurgia, o atendimento à equipe no período intraoperatório até a saída do paciente da SO e o transporte para UTI, atendendo às demandas dessa modalidade cirúrgica inovadora.

Diante de tantos avanços, novos desafios e responsabilidades, a equipe de enfermagem do CC necessita de atualização e treinamento constantes na busca de embasamento técnico-científico para o desenvolvimento de suas atividades, com vistas ao sucesso do procedimento anestésico-cirúrgico.

Nesse sentido, este relato de experiência almejou auxiliar outros hospitais e equipes perioperatórias no preparo e na realização de cirurgias cardíacas minimamente invasivas mediante assistência segura e humanizada.

\section{REFERÊNCIAS}

1. Felcar MJ, Guitti JCS, Marson CA, Cardoso RC. Fisioterapia préoperatória na prevenção das complicações pulmonares em cirurgia cardíaca pediátrica. Rev Bras Cir Cardiovasc. 2008;23(3):383-8.
2. Castro Neto JV, Melo EM, Silva JF, Rebouças LL, Correa LC, Germano AQ, et al. Procedimentos minimamente invasivos: formas direta e videoassistida no tratamento das cardiopatias. Arq Bras Cardiol. 2014;102(3):219-25. 
3. Fortunato Júnior AJ, Fernandes AG, Sesca JR, Paludo R, Paz ME, Paludo $L$, et al. Troca valvar aórtica minimamente invasiva: uma alternativa a técnica convencional. Rev Bras Cir Cardiovasc. 2012;27(4):570-82.

4. Moreira LFP, Celullari AL. Cirurgia cardíaca minimamente invasiva no Brasil. Rev Bras Cir Cardiovasc. 2011;26(4):3-5.

5. Grossi EA, Goldman S, Wolfe JA, Mehall J, Smith JM, Ailawadi G, et al. Minithoracotomy for mitral valve repair improves inpatient and postdischarge economic savings. J Thorac Cardiovasc Surg. 2014;148(6):2818-22.

6. Granov N, Kacila M, Solinas M, Glauber M. Minimally invasive mitral valve surgery through right lateral minithoracotomy--early experience of Clinical Centre of University of Sarajevo. Med Arch. 2012;66(5):357-8.

7. Pereira MCO, Lário A. Cirurgias minimamente invasivas. In: Carvalho $\mathrm{R}$ (Coord.). Enfermagem em centro de centro cirúrgico e recuperação anestésica. Barueri: Manole; 2015. p.12-6.

8. Brevidelli MM, Sertório SM. Trabalho de conclusão de curso: guia prático para docentes e alunos da área da saúde. $4^{\mathrm{a}}$ ed. São Paulo: látria; 2010.

9. Fortunato Junior AJ, Branco Filho AA, Branco A, Martins ALM, Pereira LM, Ferraz JGG, et al. Padronização da técnica para cirurgia cardíaca videoassistida: experiência inicial. Rev Bras Cir Cardiovasc. 2008;23(2):183-9.

10. Milani R, Brofman PRS, Oliveira S, Patrial Neto L, Rosa M, Lima VH, et al. Minimally invasive redo mitral valve surgery without aortic crossclamp. Rev Bras Cir Cardiovasc. 2013;28(3):325-30.

11. Jardim DP, Carvalho R. Assistência de enfermagem na sala operatória/ cirurgia segura. In: Carvalho R (Coord.). Cadernos de Especialidades Enfermagem em Centro Cirúrgico e Recuperação Anestésica. Barueri: Manole; 2015. p. 104-28.
12. Lima GF, Rinaldi PA, Catunda KA, Bianchi ERF, Bittar E. Atuação do enfermeiro na estruturação e implantação de uma sala operatória híbrida. Rev SOBECC. 2014;19(2):110-8.

13. Pancieri PA, Carvalho R, Braga ME. Aplicação do checklist para cirurgia segura: relato de experiência. Rev SOBECC. 2014;19(1):26-33.

14. Jardim DP, Coutinho RMC, Bianchi ERF, Costa ALS, Váttimo MFF. Assistência de enfermagem no período transoperatório. In: Carvalho R, Bianchi ERF (Orgs.). Enfermagem em centro cirúrgico e recuperação. $2^{\text {a }}$ ed. Barueri: Manole; 2016. p.146-59.

15. Saraiva LI, Paula MFC, Carvalho R. Úlcera por pressão no período transoperatório: ocorrência e fatores associados. Rev SOBECC. 2014;19(4):207-13.

16. Parra RLC, Giannasttasio MB, Diniz TRZ. O conhecimento dos circulantes de sala sobre a utilização do Bisturi elétrico. Rev SOBECC. 2012;17(4):24-32.

17. Dienstamann C, Caregnato RCA. Circulação extracorpórea em cirurgia cardíaca: um campo de trabalho para o enfermeiro. Rev SOBECC. 2013;18(1):35-43.

18. Fortunato Júnior JA, Pereira ML, Martins AL, Pereira DS, Paz ME, Paludo L, et al. Video-assisted cardiac surgery: 6 years of experience. Rev Bras Cir Cardiovasc. 2012;27(1):24-37.

19. Brasil. Ministério da Saúde (MS). Agencia Nacional de Vigilância Sanitária (ANVISA). Resolução da Diretoria Colegiada (RDC) nº. 307, de 14 de novembro de 2002. Altera a Resolução RDC no. 50 de 21 de fevereiro de 2002 que dispõe sobre o regulamento Técnico para planejamento, programação, elaboração e avaliação de projetos físicos de estabelecimentos assistenciais de saúde. [acesso em 14 out 2015]. Disponível em: www.cvs.saúde.sp.gov.br

20. Gelape CL. Infecção do sítio operatório em cirurgia cardíaca. Arq Bras Cardiol. 2007;89(1):3-9. 